\title{
Extracting chemical reactions from text using Snorkel
}

Emily K. Mallory ${ }^{1}$, Matthieu de Rochemonteix ${ }^{2}$, Alex Ratner ${ }^{3}$, Ambika Acharya ${ }^{3}$, Chris Re ${ }^{3}$, Roselie A. Bright ${ }^{4}$ and Russ B. Altman ${ }^{5^{*}}$ (D)

\author{
* Correspondence: russ.altman@ \\ stanford.edu \\ ${ }^{5}$ Departments of Medicine, \\ Genetics, Bioengineering, and \\ Biomedical Data Science, Stanford \\ University, Stanford, CA, USA \\ Full list of author information is \\ available at the end of the article
}

\begin{abstract}
Background: Enzymatic and chemical reactions are key for understanding biological processes in cells. Curated databases of chemical reactions exist but these databases struggle to keep up with the exponential growth of the biomedical literature.

Conventional text mining pipelines provide tools to automatically extract entities and relationships from the scientific literature, and partially replace expert curation, but such machine learning frameworks often require a large amount of labeled training data and thus lack scalability for both larger document corpora and new relationship types.

Results: We developed an application of Snorkel, a weakly supervised learning framework, for extracting chemical reaction relationships from biomedical literature abstracts. For this work, we defined a chemical reaction relationship as the transformation of chemical A to chemical B. We built and evaluated our system on small annotated sets of chemical reaction relationships from two corpora: curated bacteria-related abstracts from the MetaCyc database (MetaCyc_Corpus) and a more general set of abstracts annotated with MeSH (Medical Subject Headings) term Bacteria (Bacteria_Corpus; a superset of MetaCyc_Corpus). For the MetaCyc_Corpus, we obtained $84 \%$ precision and $41 \%$ recall (55\% F1 score). Extending to the more general Bacteria_Corpus decreased precision to $62 \%$ with only a four-point drop in recall to $37 \%$ (46\% F1 score). Overall, the Bacteria_Corpus contained two orders of magnitude more candidate chemical reaction relationships (nine million candidates vs 68,0000 candidates) and had a larger class imbalance ( $2.5 \%$ positives vs $5 \%$ positives) as compared to the MetaCyc_Corpus. In total, we extracted 6871 chemical reaction relationships from nine million candidates in the Bacteria_Corpus.
\end{abstract}

Conclusions: With this work, we built a database of chemical reaction relationships from almost 900,000 scientific abstracts without a large training set of labeled annotations. Further, we showed the generalizability of our initial application built on MetaCyc documents enriched with chemical reactions to a general set of articles related to bacteria.

Keywords: Text mining, Chemical reactions, Snorkel, Curation, Database

\section{$\triangle B M C$}

(c) The Author(s). 2020 Open Access This article is licensed under a Creative Commons Attribution 4.0 International License, which permits use, sharing, adaptation, distribution and reproduction in any medium or format, as long as you give appropriate credit to the original author(s) and the source, provide a link to the Creative Commons licence, and indicate if changes were made. The images or other third party material in this article are included in the article's Creative Commons licence, unless indicated otherwise in a credit line to the material. If material is not included in the article's Creative Commons licence and your intended use is not permitted by statutory regulation or exceeds the permitted use, you will need to obtain permission directly from the copyright holder. To view a copy of this licence, visit http://creativecommons.org/licenses/by/4.0/. The Creative Commons Public Domain Dedication waiver (http://creativecommons.org/publicdomain/zero/1.0/) applies to the data made available in this article, unless otherwise stated in a credit line to the data. 


\section{Background}

Enzymes and the reactions they catalyze are key for understanding how small molecules are processed in the cell. In particular, chemical reactions that occur in the human gut microbiome can shed light on drug mechanism of action and metabolism when these reactions transform drugs or other bio-active small molecules. Since unintentional drug transformations in the human gut can affect drug response and side effects in patients [1], understanding the space of chemical reactions in bacteria is necessary for predicting and cataloging enzymatic transformations of drugs in the human gut microbiome.

Databases such as MetaCyc [2] and KEGG (Kyoto Encyclopedia of Genes and Genomes) [3] contain high quality pathways with metabolic reactions that are manually annotated by human experts. However, manual human annotation restricts the coverage and growth of the database with respect to the biomedical literature. This inability to scale to larger and larger corpora is a limiting factor in large data-driven studies. On the other hand, the biomedical literature is the single best source of known metabolic reactions across all organisms. While rich in information, the computationally inaccessible nature of literature text presents a challenge for relationship extraction.

Extraction of chemical reactions from text requires the extraction of chemical entities and the relationship between them as described in a sentence or paragraph. In natural language processing, the task of entity extraction is referred to as named entity recognition and the task of connecting two entities in a defined relationship is relationship extraction. For biological datasets, text mining tools exist to extract protein-protein [4] and other biomedical associations [5]. While PubTator from the National Center for Biotechnology Information [6] (using the method tmChem [7]) extracts and releases datasets of chemical entities extracted from abstract sentences, to our knowledge no method exists for extracting the primary transformations occurring in chemical reactions from text. In order to build a database of chemical reactions from text, a text mining application framework is needed for rapid development with high accuracy, usability for nontechnical users, and corpus size scalability in order to enable downstream analyses using the resulting extracted relationship database.

Recent advances in natural language processing and word embeddings have introduced new approaches for relationship extraction. Self-supervised word embedding approaches such as word2vec [8] and BERT (Bidirectional Encoder Representations from Transformers) [9] construct vector representations of words based on the context around a word in a given corpus. BERT is a language representational model that has been applied to a number of NLP (natural language processing) tasks, such as named entity recognition and relationship extraction. Building on the original BERT model, Lee et al. released BioBERT - a BERT model trained on biomedical literature from PubMed and PubMed Central [10]. BioBERT achieved between 75 and 77\% precision for the task of detecting protein-chemical relationships, a task included in BioCreative VI $[11,12]$. While Bio-BERT demonstrated improved performance for relationship extraction of protein-chemical relationships, amongst other relationships, lack of accessible text mining tools and frameworks for domain experts remains a challenge. Additionally, although community challenges have enabled the BioNLP (biomedical natural language processing) community to solve some pressing extraction tasks, a lack 
of labeled datasets has limited the applicability of these tools to other tasks in within subdomains of biology and medicine.

Snorkel is a framework for creating large training datasets and streamlining the typical natural language processing extraction pipeline for entities and relationships from text $[13,14]$. The main advantage for Snorkel is that it does not require hand-labeled datasets that may need to be relabeled, extended, or disregarded when a task or schema changes. Instead, the user only has to focus on designing relevant labeling functions to automatically assign noisy labels. These labeling functions can then be reused for updating or repurposing the literature corpus. As a result, the Snorkel framework allows very fast prototyping and good scalability to large datasets.

Users interact with Snorkel using pre-defined functions for parsing text, detecting candidate entities or relationships (e.g., pair of two entities co-occurring in a sentence), assigning training labels, extracting text-based features, and training a machine learning model for the final prediction. Importantly, users focus primarily on developing labeling functions and not constructing large hand-labeled training sets as found in typical machine learning pipelines. These labeling functions encode basic rules for automatically labeling noisy training examples for a combination of generative and discriminative machine learning models in Snorkel. The final output of Snorkel is a binary prediction of a true relationship for each candidate relationship.

The focus of databases such as MetaCyc [2] is high-quality expert curation (in MetaCyc's case, enzymatic reactions and pathways) at the expense of speed and coverage of biomedical literature. Preliminary results of applying Snorkel to multiple biomedical entity and relationship tasks demonstrated Snorkel's fast prototyping and scalability for different biomedical tasks. In this work, we built a Snorkel application to extract chemical reaction relationships from the biomedical literature in a higher-throughput and more scalable approach.

\section{Methods}

In this work, we built a knowledge base construction application, using the Snorkel framework, for extracting chemical reaction relationships from biomedical literature abstracts. The Snorkel pipeline is depicted in Fig. 1 and described below. In summary, our application takes a corpus of texts as an input and outputs all co-occurring entity pairs with a binary prediction of a true relationship. Using the Snorkel framework, we primarily focused on two tasks: extracting candidate entities and relationships and designing labeling functions. These labeling functions allow us to apply noisy labels to candidate relationships without labeling large training sets by hand. We next used these labeling functions to train a combination of generative and discriminative machine learning models that generalize beyond the initial noisy labels.

\section{Datasets}

MetaCyc_Corpus

We built and evaluated the Snorkel application using a corpus of 1799 PubMed abstracts curated for enzymatic chemical reactions in bacteria by MetaCyc [2] in version 20.0 (downloaded August 2016). We parsed the corpus using the Snorkel pipeline and the SpaCy parser [15] to detect sentences, word tokens, parts-of-speech tags and 


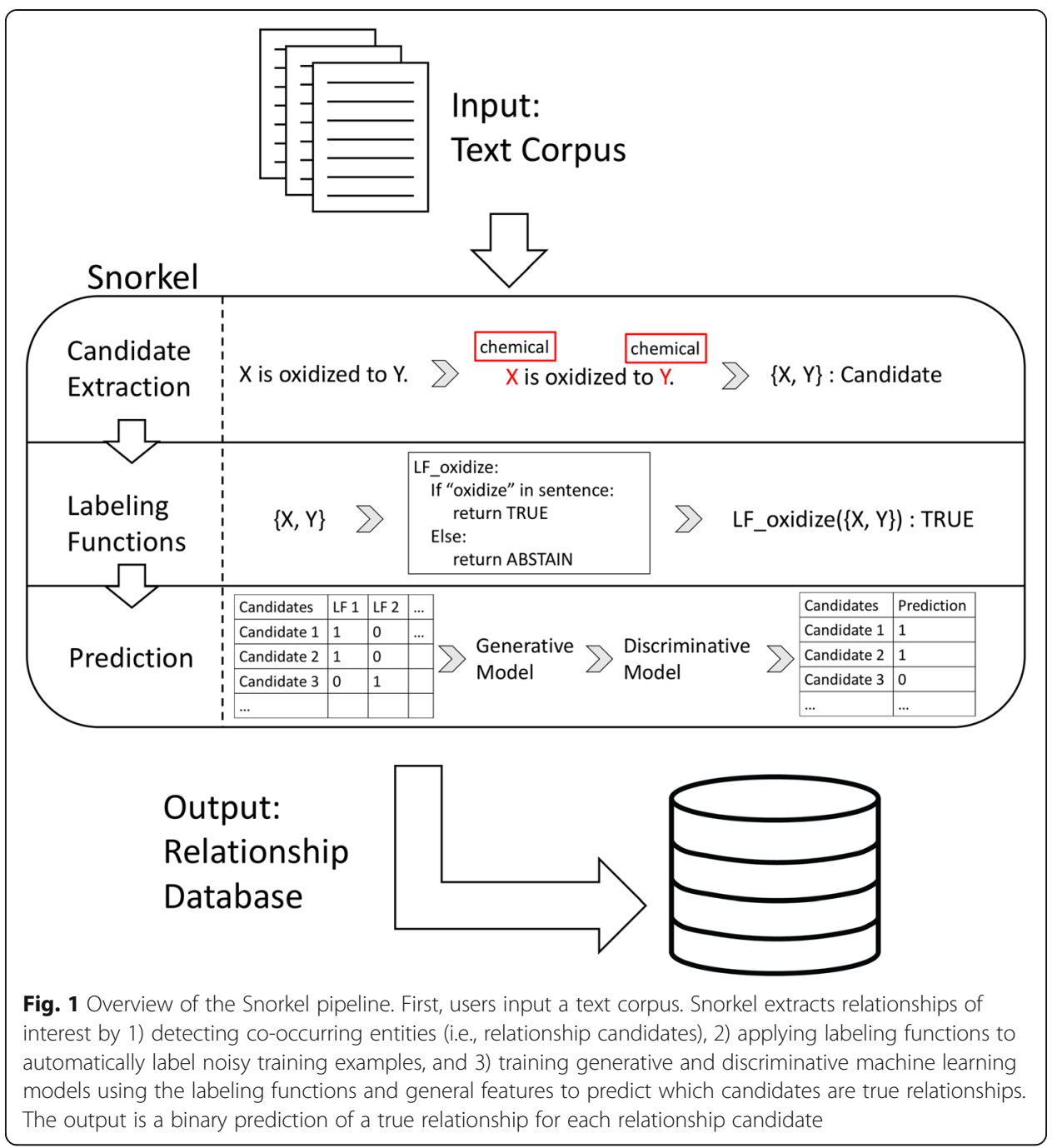

dependency graphs. We included the titles of the abstracts in the database. In addition, we included PubTator Chemical annotations [6] for all abstracts in the corpus. We refer to this corpus as the MetaCyc_Corpus.

\section{Bacteria_Corpus}

To generalize and expand the MetaCyc_Corpus, we built a second dataset using the following PubMed query:

bacteria [MeSH Terms] AND has abstract [filter] AND English [language]

This query included all abstracts in English, annotated with the MeSH term "bacteria", as of Feb 2018. We filtered these abstracts to the subset containing PubTator Chemical annotations (retrieved March 2018). From the 880,134 abstracts returned from the Pubmed query, we included 873,237 abstracts with PubTator Chemical annotations in the abstract corpus. We refer to this corpus as the Bacteria_Corpus. 
To facilitate comparison of the results between the two datasets, we added all the documents from the MetaCyc_Corpus that were available in the PubTator dump to the Bacteria_Corpus.

\section{Candidate extraction}

The primary task of relationship extraction in Snorkel is to extract pairs of entities that fit some interaction or relationship definition. This relationship definition defines a candidate relationship, a pair of entities that may participate in a true relationship. Importantly, this definition should have high recall for true relationships but may have low precision. For the task of extracting chemical reaction relationships, we want to define a relationship definition to capture the majority of chemical reactions present in literature abstracts with a focus on recall.

The following is an example sentence describing a chemical reaction:

$\mathrm{X}$ is converted into $\mathrm{Y}$ in the absence of $\mathrm{Z}$.

There is one true chemical reaction in this sentence: $\mathrm{X}$ is converted into $\mathrm{Y}$. In this example, the task of the Snorkel pipeline is to extract the pair of words (X, Y).

We define a chemical reaction relationship candidate as an ordered pair of distinct chemicals co-occurring in a sentence. In our example, there are three chemicals in the sentence: $X, Y$, and $Z$. Therefore, there are six candidate chemical reactions: $(X, Y),(X$, $\mathrm{Z})$, (Y, X), (Y, Z), (Z, X), and (Z, Y).

We performed candidate extraction on both datasets using Chemical entity tags from PubTator and a candidate extraction workflow with objects and functions built into Snorkel. In summary, we scanned sentences in both corpora for co-occurring PubTator chemical entities to create candidate relationships. As described above, we created two candidate relationships for every pair of entities to capture the correct order of the potential chemical reaction. This extraction procedure yielded a large number of candidates (for a sentence with $n$ chemicals, we have $n(n-1)$ candidates). Candidate counts are reported in Table 1.

It is important to note that the Bacteria_Corpus is 485 times larger than the MetaCyc_Corpus in terms of the number of documents, but it is only 132 times larger in terms of candidates. The documents in the MetaCyc_Corpus have on average 3.69 more candidates and are understandably enriched for sentences that discuss chemical reactions.

\section{Learning procedure}

Candidate extraction produces a set of candidate chemical reaction relationships. During learning, we trained machine learning models to predict which candidates were true chemical reactions (Fig. 1, steps 2 and 3). We first designed labeling functions to

Table 1 Number of abstracts and candidates in MetaCyc_Corpus and Bacteria_Corpus

\begin{tabular}{lll}
\hline Corpus & Abstracts & Candidates \\
\hline MetaCyc_Corpus & 1799 & 67,922 \\
Bacteria_Corpus & 873,237 & $8,936,941$ \\
\hline
\end{tabular}


automatically label noisy training examples. Next, we trained a generative model to learn the inaccuracies of the labeling functions. Finally, we trained a discriminative model using the scores from the generative model as training labels to predict true chemical reactions.

\section{Labeling functions}

Labeling functions have three important features:

- Labeling functions are context-aware: they may rely on sentence or document-level patterns, use the entity tags or labels, or even use external information.

- Labeling functions can be very general and correspond to a weak signal, or they can be very specific and handle special, uncommon cases.

- Labeling functions have 3 possible outputs: TRUE, FALSE, or ABSTAIN.

We designed unipolar labeling functions, that only have two possible outputs. The positive labeling functions select either TRUE or ABSTAIN, while the negative labeling functions select either FALSE or ABSTAIN. These unipolar labeling functions deviate from prior work [14] in order to handle large class imbalances. Importantly, labeling functions must only provide noisy information about the likelihood of a chemical reaction and need not be individually very predictive. For the MetaCyc_Corpus, we designed five positive labeling functions and 11 negative functions. Example positive and negative labeling functions are detailed in Table 2. Descriptions of all labeling functions are listed in Supplemental Data Section 1.7.

Because the Bacteria_Corpus is over 100 times larger than the MetaCyc_Corpus and had a larger variety of candidates, we designed an extra set of labeling functions to capture the complexity and diversity of this dataset. Table 3 contains examples of additional labeling functions for the Bacteria_Corpus. We added five additional negative labeling functions and one positive, to obtain an overall set of 22 labeling functions for the Bacteria_Corpus. For LF_metacyc, we used <substrate, product> pairs from enzymatic reactions from MetaCyc (version 20.0). We limited these reactions to include only 1:1 substrate-product transformations, after filtering proton acceptors and donors, water molecules, and hydrogen atoms.

\section{Generative model}

After applying the labeling functions to the candidate relationships, we trained a generative model to learn the inaccuracies of the labeling functions and assign a

Table 2 Example labeling functions for the MetaCyc corpus

\begin{tabular}{|c|c|}
\hline $\begin{array}{l}\text { Example labeling } \\
\text { function }\end{array}$ & Description \\
\hline LF_keyword_context & $\begin{array}{l}\text { If there is a word of a given list, such as reduce, oxidize, transform, or afford between the } \\
\text { two words, we label TRUE }\end{array}$ \\
\hline LF_sep_verb & If the chemicals are separated by a verb, we label TRUE \\
\hline LF_argument_order & If the candidate product is before the candidate substrate, we label FALSE \\
\hline LF_followed_ase & If one of the chemicals is followed by a word that ends with "ase", we label FALSE \\
\hline LF_sep_or & If the chemicals are separated by the word or, we label FALSE \\
\hline
\end{tabular}


Table 3 Examples of additional labeling functions on the Bacteria_Corpus

\begin{tabular}{ll}
\hline $\begin{array}{l}\text { Example labeling } \\
\text { function }\end{array}$ & Description \\
\hline LF_metacyc & If the chemical reaction is already in the MetaCyc curated database, we label TRUE \\
LF_chemical_elements & If one of the chemicals is a chemical element, we label FALSE \\
LF_group & If there is a close mention of a functional chemical group, we label FALSE \\
LF_treatment & If there is mention of keywords frequently associated with clinical trials, we label \\
& FALSE
\end{tabular}

probabilistic score to each candidate. From the labeling function step, the labels (from the labeling functions) for the extracted candidates form a sparse matrix of 0,1 and - 1 (ABSTAIN, TRUE, and FALSE, respectively). The purpose of the generative model is to infer an underlying distribution from which those labels have been sampled, and to use this model to score all the candidates from 0 to 1 ( 0 being a negative relationship and 1 a positive relationship). We refer to these scores as the training marginals. The training marginals formed the training example labels to the discriminative model (described below).

We used different versions of the generative model implementation for the MetaCyc_Corpus and Bacteria_Corpus. The current released version of Snorkel uses a Gibbs Sampling method, that has been thoroughly tested and evaluated by the Snorkel developers [13, 14]. We used this version for the MetaCyc_Corpus. However, this implementation is not robust to unbalanced sets of unipolar labeling functions and heavy class imbalance in the dataset.

Because the Bacteria_Corpus has a very high class imbalance and unbalanced set of unipolar labeling functions, we used a new class-conditional matrix completion based approach of the generative model that is much more robust to the imbalance [16]. As this implementation is not needed on the MetaCyc_Corpus, we preferred keeping the Gibbs Sampling implementation when possible (see Supplemental Section 1.6 for comparison of the two generative model approaches).

\section{Discriminative model}

Whereas the generative model provides probabilistic labels for each candidate using the labeling functions, the discriminative model generalizes beyond the labeling functions by using the training marginals as training example labels.

We trained a discriminative model using the training marginals from the generative model as training labels. The discriminative model is a logistic regression model that uses a set of default NLP features (n-grams, etc...) computed on the training set. We trained the discriminative model using the sigmoid cross-entropy loss for binary classification, with an elastic net penalty. Before training the discriminative model, we resampled the dataset (see Supplemental Section 1.1 for the detailed procedure and Supplemental Section 1.5 for comments). The hyperparameters for the discriminative model and the resampling were chosen using a grid search on a development set with a small number of labeled candidate chemical reaction relationships (described in Section Evaluation Framework).

\section{Evaluation framework}

One of the main advantages of the Snorkel pipeline is that we do not need to manually annotate training data and instead rely on the labeling functions and a small annotated 
development set and held-out test set. For performance evaluation, as well as labeling function and model design, we curated chemical reaction relationship candidates in a subset of abstracts from the two chemical reaction corpora.

\section{Evaluation datasets for MetaCyc_Corpus}

To achieve proper reproducibility and evaluation of the performance, we split the MetaCyc_Corpus into three distinct splits of the data.

- MetaCyc_Train: constituted the majority of the abstracts. We trained the models on this split only. However, unlike the training sets in usual machine learning pipelines, the candidates have not been manually annotated.

- MetaCyc_Dev: a small development subset of the initial corpus used to tune the model parameters using gridsearch and design the labeling functions

- MetaCyc_Test: a subset of the corpus held out from model training and development to evaluate the out-of-sample model performance.

We randomly sampled abstracts without replacement from the full corpus to create MetaCyc_Dev and MetaCyc_Test and curated the associated candidates. Table 4 reports the number of abstracts, candidates, and positive examples for the train, development and test sets.

\section{Evaluation datasets for Bacteria_Corpus}

We built the Bacteria_Corpus by extending the MetaCyc_Corpus. The extension of the dataset created a need to have a new test set to evaluate the performance of the models on the updated task. However, we kept MetaCyc_Test as a test set to have an estimator of the model performance on the MetaCyc_Corpus (that is a subset of the Bacteria Corpus). It is also necessary to keep MetaCyc_Dev, as it has been used to design a majority of the labeling functions.

As a result, the Bacteria_Corpus experiment relied on a four-split architecture, divided as follows:

- Bacteria_Train: the training set, constituted the majority of the abstracts

- Bacteria_Test: a subset of the corpus held out from model training and development to evaluate the out-of-sample model performance

- Bacteria_Dev: MetaCyc_Dev, augmented with 200 abstracts randomly sampled from the new documents in the Bacteria_Corpus to gridseach the models and design the labeling functions.

- MetaCyc_Test: included with no change.

Table 4 Sizes and gold label statistics of the three splits for the MetaCyc_Corpus

\begin{tabular}{llllll}
\hline Split & Abstracts & Candidates & Positives & Docs w. candidates & Docs w. positives \\
\hline MetaCyc_Train & 1753 & 65,398 & - & 1544 & - \\
MetaCyc_Dev & 23 & 1292 & 60 & 23 & 16 \\
MetaCyc_Test & 23 & 1232 & 51 & 23 & 15 \\
\hline
\end{tabular}


We randomly sampled abstracts without replacement from the full corpus to create Bacteria_Test and Bacteria_Dev and curated all candidates in the sampled abstracts. Table 5 reports the number of abstracts, candidates, and positive examples for the different splits of the Bacteria_Corpus. The 400 documents added to Bacteria_Test and Bacteria_Dev were randomly sampled from the documents that were not already in MetaCyc_Dev or MetaCyc_Test. Therefore, we developed the final models on Bacteria Dev and evaluated performance on both Bacteria_Test and MetaCyc_Test.

Due to minor updates in the candidate extraction process, and in the PubTator chemical tags, there is $<1 \%$ discrepancy in the number of candidates extracted between the MetaCyc and the Bacteria experiments for the 1791 abstracts that are in both corpus.

\section{Evaluation metrics}

We evaluated the performance of three prediction models: majority voting of the labeling functions, generative model only, and discriminative model using the training marginals from the generative model (henceforth referred to as discriminative model for simplicity). We computed precision, recall, and $F_{1}$ score using the development and held-out test sets for MetaCyc_Corpus and Bacteria_Corpus.

Precision $: P=\frac{T P}{T P+F P}$

Recall $: R=\frac{T P}{T P+F N}$

$F_{1}$ score $: \frac{2 P * R}{P+R}$

Where:

- TP (True Positives): Positive examples, correctly classified

- TN (True Negatives): Negative examples, correctly classified

- FP (False Positives): Negative examples, misclassified

- FN (False Negatives): Positive examples, misclassified

We also used the $F_{\beta}$ score to select the models in the gridsearch, as it allows to shift the precision-recall trade-off towards more precision or more recall if needed.

The $\mathrm{F}_{\beta}$ score is defined as $\left(1+\beta^{2}\right) \frac{P * R}{\beta^{2} P+R}$.

Table 5 Sizes and gold label statistics of the splits for the Bacteria_Corpus

\begin{tabular}{llllll}
\hline Split & Abstracts & Candidates & Positives & Docs w. candidates & Docs w. positives \\
\hline Bacteria_Train & 872,591 & $8,928,937$ & - & 417,404 & - \\
Bacteria_Test & 200 & 2398 & 43 & 96 & 13 \\
Bacteria_Dev & 223 & 2806 & 69 & 110 & 22 \\
MetaCyc_Test & 23 & 1212 & 49 & 23 & 15 \\
\hline
\end{tabular}




\section{Results}

We built and evaluated the Snorkel application for extracting chemical reaction relationships from two datasets: MetaCyc_Corpus and Bacteria_Corpus.

\section{Comparison of labeling function coverage}

An important component of the labeling function design process is to analyze the statistics of the different labeling functions. Table 6 provides example labeling functions with proportion coverage, overlaps, and conflicts in the MetaCyc_Corpus and the Bacteria_Corpus. For MetaCyc_Corpus, there are both very wide labeling functions and very precise ones. For example, LF_argument_order has a 0.50 coverage or proportion of candidates labeled by the labeling function because of its generality. However, LF_ keyword_context only labels rare candidates and therefore has a low coverage. The coverage of the labeling functions initially designed for the MetaCyc_Corpus dropped when we applied the labeling functions to the Bacteria_Corpus. For example, the coverage of LF_followed_ase dropped from 0.18 to 0.015 .

\section{Evaluation on the MetaCyc_Corpus}

We evaluated Snorkel for the task of extracting chemical reaction relationships from text. Table 7 contains the evaluation result using majority voting, generative model, and discriminative model on the MetaCyc_Corpus. The generative model performed similarly to majority voting. However, the discriminative model brought a significant lift of the performance and increased precision from 0.79 to 0.84 for the discriminative model. In addition, there was a 19 point increase in recall from 0.22 to 0.41 .

\section{Evaluation on the Bacteria_Corpus}

We evaluated Snorkel for the task of extracting chemical reaction relationships from text. Table 8 contains the evaluation result using majority voting, generative model, and discriminative model on the Bacteria_Corpus using MetaCyc_Test. We used a threshold of 0.50 on the final score given by the discriminative model to issue predictions.

Table 6 Labeling Function metrics for MetaCyc_Corpus and Bacteria_Corpus. Coverage refers to the proportion of candidates labeled with the labeling function. Overlaps refers to the proportion of candidates labeled with another labeling function. Conflicts refers to proportion of candidates labeled with an opposing labeling function

\begin{tabular}{lllllllll}
\hline & \multicolumn{3}{l}{ MetaCyc_Corpus } & & \multicolumn{3}{l}{ Bacteria_Corpus } \\
\cline { 2 - 3 } Labeling function & Coverage & Overlaps & Conflicts & & Coverage & Overlaps & Conflicts \\
\hline LF_keyword_context & 0.005963 & 0.002110 & 0.001361 & & 0.001902 & 0.001750 & 0.001719 \\
LF_sep_verb & 0.000933 & 0.000291 & 0.000092 & & 0.001252 & 0.001146 & 0.001137 \\
LF_argument_order & 0.500000 & 0.238234 & 0.005520 & & 0.499939 & 0.470721 & 0.016873 \\
LF_followed_ase & 0.180954 & 0.155800 & 0.001697 & & 0.015969 & 0.015838 & 0.000966 \\
LF_sep_or & 0.006453 & 0.003333 & 0.000000 & & 0.006702 & 0.006399 & 0.000526 \\
LF_metacyc & - & - & - & & 0.031805 & 0.030915 & 0.030823 \\
LF_chemical & - & - & - & - & 0.130835 & 0.130539 & 0.004871 \\
LF_treatment & - & - & - & & 0.029490 & 0.028674 & 0.000609 \\
\hline
\end{tabular}


Table 7 Evaluation results for MetaCyc_Corpus. We evaluated three models: majority voting, generative model, and discriminative model

\begin{tabular}{lllll}
\hline Model & Coverage & Precision & Recall & F1 Score \\
\hline Majority voting & 0.73 & 0.79 & 0.22 & 0.34 \\
Generative model & 0.73 & 0.79 & 0.22 & 0.34 \\
Discriminative model & $\mathbf{1 . 0 0}$ & $\mathbf{0 . 8 4}$ & $\mathbf{0 . 4 1}$ & $\mathbf{0 . 5 5}$
\end{tabular}

The generative model brings a significant lift on the recall for Bacteria_Corpus, increasing recall 12 points from 0.20 to 0.33 while preserving the precision. The discriminative model changes the precision-recall tradeoff towards a more balanced prediction, that lifts the $F_{1}$ score by six points for the discriminative model compared to the generative model. While precision decreased to 0.50 , recall increased to 0.61 .

In total, we extracted 6871 chemical reaction relationships from almost nine million candidates in Bacteria_Corpus. This total includes both chemical reaction relationships from MetaCyc_Corpus and Bacteria_Corpus.

\section{Discussion}

We designed a Snorkel application to construct a chemical reaction database from text using a small corpus of abstracts enriched with enzymatic chemical reactions and extended this application to a more general corpus of almost a million abstracts broadly related to bacteria. The ability to generate relationship data from domain-specific literature is essential to creating rich datasets for improving large data-driven tasks. We defined a chemical reaction relationship for extraction; however, we note that our approach is much more easily modified to select different (e.g. more specific) relation types of interest, as this only requires modifying code, rather than relabeling datasets by hand as in traditional approaches. In addition, our results can be used to develop Snorkel extensions to increase accuracy for complex chemical reaction and chemistry literature.

This framework substantially changes how one approaches text mining applications and development, and considerably speeds up the extractor creation process. Importantly, the focus on designing labeling functions in Snorkel allows for quicker development time and scalability to larger and more general datasets. We developed 16 labeling functions for labeling almost 68,000 candidates from MetaCyc_Corpus and added only six new labeling functions to label almost nine million candidates from Bacteria_Corpus. These labeling functions included high precision/low recall functions such as if two chemicals are separated by a sequence of words that contains oxidized to, reduced to, conversion to, label candidate TRUE. This labeling function labels candidates that are likely correct (i.e., marginal probability close to 1 ) but will miss many candidates that do not have straightforward syntax describing the relationship. Other

Table 8 Evaluation results on the Bacteria_Corpus, using a 0.5 threshold. We evaluated three models: majority voting, generative model, and discriminative model

\begin{tabular}{lllll}
\hline Model & Coverage & Precision & Recall & F1 Score \\
\hline Majority voting & 0.92 & $\mathbf{1 . 0 0}$ & 0.20 & 0.34 \\
Generative model & 0.94 & $\mathbf{1 . 0 0}$ & 0.33 & 0.49 \\
Discriminative model & $\mathbf{1 . 0 0}$ & 0.50 & $\mathbf{0 . 6 1}$ & $\mathbf{0 . 5 5}$ \\
\hline
\end{tabular}


labeling functions will be less specific but label many candidates either True or False. For example, a labeling function that returns True if the second chemical follows the first chemical in the sentence labeled $50 \%$ of all candidates in both MetaCyc_Corpus and Bacteria_Corpus. However, a labeling function that returns True if a verb separates two chemicals in a sentence labeled a smaller subset of the data $(0.09$ and $0.1 \%$ for MetaCyc_Corpus and Bacteria_Corpus, respectively). While precision decreased compared to MetaCyc_Corpus, Bacteria_Corpus included a more diverse set of documents (all documents related to bacteria using MeSH terms) which in turn included a more diverse chemical set. Thus while Bacteria_Corpus included more false positive chemical reaction relationships, we increased recall substantially to capture more true chemical reaction relationships. The boost in recall from generative to discriminative model allowed for more generalization in the Bacteria_Corpus. The focus on precision vs recall is a design decision that depends partially on downstream application use.

From almost nine million candidates in Bacteria_Corpus, we extracted 6871 chemical reaction relationships. These reactions included simple substrate-product pairs. For example, we extracted the reaction gluconic acid to ethanol from the sentence "We report on engineering Escherichia coli to produce ethanol at high yield from gluconic acid [17]." We also extracted reactions where multiple substrates and/or products were mentioned in the sentence. For example, Snorkel extracted the hydrolysis of naproxen nitrile to S-naproxen from the sentence "Enantioselective hydrolysis of racemic naproxen nitrile and naproxen amide to S-naproxen by new bacterial isolates [18]." The sentence "These results suggest that HMF can be metabolically activated to an allylic sulfuric acid ester which may play a role as an ultimate electrophilic metabolite in toxification of the parent compound in vivo." from [19] presents an interesting example. Here, we extracted HMF goes to allylic sulfuric acid ester. While we know the starting compound, we do not know the exact product. However, these are important cases to capture since chemistry literature can be vague on the chemical structure and name depending on when the article was written.

While we extracted chemical reaction relationships from clear substrate to product transformations, Snorkel struggled to correctly detect negative reactions when cooccurring with a positive reaction. For example, the sentence "Only D-cysteine but not L-cysteine was converted by D-CDes to pyruvate, H2S, and NH3." [20] includes multiple potential substrate and product pairs. However, only one of the potential substrates is a true substrate in the described reaction (D-cysteine). Snorkel extracted all pairs of the potential substrates D-cysteine and L-cysteine with the products pyruvate, $\mathrm{H} 2 \mathrm{~S}$, and NH3. The resulting extracted reactions include the correct reaction pairs (Dcysteine, pyruvate), (D-cysteine, H2S), and (D-cysteine, NH3) but also incorrect reaction pairs (L-cysteine, pyruvate), (L-cysteine, H2S), and (L-cysteine, NH3). Thus in this sentence, $50 \%$ of extractions were incorrect. It is important to note that none of the substrates were extracted together in an incorrect or false chemical reaction, and neither were the potential products. Additional curated candidates in the development and test sets, along with further development of the labeling functions, would provide additional signal to detect negative candidates co-occurring with positive candidates correctly.

Class imbalance within and between the two corpora was a key challenge of this work. An estimation of the proportion of positive candidates can be done based on the 
number of positive candidates in MetaCyc_Dev (reported in Table 4). This reveals a high class imbalance (5\%). Table 4 also shows that $88 \%$ of the documents have at least a candidate (decreases to $47 \%$ in Bacteria_Corpus). However, the labeled subsets suggest that only $67 \%$ of the documents that have a candidate have a positive candidate. The class imbalance is higher in the Bacteria_Corpus. The labeled subsets suggest that for the Bacteria_Corpus, only $13 \%$ of the documents with a candidate have a positive candidate, which is significantly lower than the MetaCyc_Corpus, especially taking into account that the MetaCyc_Corpus is included in the Bacteria_Corpus. The MetaCyc Corpus is composed of articles curated for enzymatic reactions and is thus enriched for these types of chemical reactions (see Table 1). The overall proportion of positive candidates drops to roughly $1.5 \%$ on the Bacteria_Corpus. This makes the class imbalance challenge even more difficult compared to MetaCyc_Corpus.

To address the class imbalance problem, we applied a resampling procedure to subsample the training data used in the discriminative model (described in Supplemental Methods). Additional experiments performed showed that the optimal results were obtained by training on approximately $10 \%$ of the dataset (see Supplemental Data). We found that the negative examples were very similar to one another and did not bring much diversity in the training set.

One of the hurdles for more domain and subdomain-specific extraction tools remains the challenge-focused aspect of relationship extraction development. There are wellcurated training and evaluation datasets for chemical-disease and protein-protein relationships, amongst others. These datasets arose from community challenges to develop state-of-the-art methods for biomedical entity and relationship extraction (e.g., BioCreative VI) [11]. These community-drive challenges are critical for moving the field forward in terms of method development and solving specific extraction tasks. However, there remains a need for accessible text mining application and/or tool development for domain experts. These users have specific tasks relevant to their research and subfield of biology and medicine. Snorkel allows these domain experts to develop applications for their needs. In this work, we developed a Snorkel application for the task of chemical reaction relationship extraction and demonstrated the labeling function development process for future Snorkel applications.

\section{Conclusion}

We developed a Snorkel application for extraction chemical reaction relationships from literature abstracts using an enriched corpus of chemical reactions and extended the application to a larger and diverse set of abstracts related to bacteria. In total, we extracted chemical reaction relationships from nearly 900,000 abstracts from PubMed related to bacteria. In this work, we showed the first biological application of the Snorkel infrastructure and the scalability of the Snorkel pipeline to very large datasets. This work enables development of future Snorkel extraction tasks and downstream prediction analyses based on enzymatic reaction data.

\section{Supplementary information}

Supplementary information accompanies this paper at https://doi.org/10.1186/s12859-020-03542-1.

Additional file 1. "Extracting Chemical Reactions from Text using Snorkel Supplemental Data". File contains supplemental methods and results for the main manuscript. 
Additional file 2. Tab-delimited file contains the list of 6871 extracted bacterial chemical reactions. The supplemental data file contains NCBI PubMed identifiers (i.e., pmids), sentence number, extracted chemicals and character positions in the abstract text, and training marginals for the prediction algorithm. All extracted chemical reactions are predicted as chemical reactions from the discriminative model.

\section{Abbreviations}

BERT: Bidirectional Encoder Representations from Transformers; BioNLP: Biomedical natural language processing; KEGG: Kyoto Encyclopedia of Genes and Genomes; MeSH: Medical Subject Headings; NLP: Natural language processing

\section{Acknowledgments}

We are grateful for the comments of Adam Lavertu on the final manuscript.

\section{Authors' contributions}

$E K M, R A B$, and RBA designed the Snorkel application goals and approach. EKM, MdR, and AA designed the labeling functions. EKM and MdR developed and refined the snorkel models. AR and CR helped tune the Snorkel model and provided application development feedback and guidance. EKM and AA developed evaluation datasets. EKM and MdR analyzed model performance and results. EKM and MdR wrote the manuscript. AR, MdR, RAB, and RBA provided substantial contributions during manuscript writing and revision. All authors read ad approved the final manuscript.

\section{Funding}

This work was made possible by grant U01FD004979 from the Food and Drug Administration, which supports the UCSF-Stanford Center for Excellence in Regulatory Science. Its contents are solely the responsibility of the authors and do not necessarily represent the official views of the US Department of Health and Human Services or FDA. EKM is supported by NIH NRSA F31 LM012354. RBA is supported by NIH LM05652. Some of this work used the XStream computational resource, supported by the National Science Foundation Major Research Instrumentation program (ACl1429830). Some of the computing for this project was performed on the Sherlock cluster. We would like to thank Stanford University and the Stanford Research Computing Center for providing computational resources and support that contributed to these research results. The funding bodies played no role in the design of the study and collection, analysis, and interpretation of data and in writing the manuscript.

AR and CR gratefully acknowledge the support of DARPA under Nos. FA87501720095 (D3M) and FA86501827865 (SDH), NIH under No. N000141712266 (Mobilize), NSF under Nos. CCF1763315 (Beyond Sparsity), CCF1563078 (Volume to Velocity), and DGE-114747 (NSF GRF), ONR under No. N000141712266 (Unifying Weak Supervision), the Moore Foundation, NXP, Xilinx, LETI-CEA, Intel, Google, NEC, Toshiba, TSMC, ARM, Hitachi, BASF, Accenture, Ericsson, Qualcomm, Analog Devices, the Okawa Foundation, and American Family Insurance, and members of the Stanford DAWN project: Intel, Microsoft, Teradata, Facebook, Google, Ant Financial, NEC, SAP, and VMWare.

AR is supported by the Stanford Interdisciplinary Graduate and Bio-X fellowship.

The U.S. Government is authorized to reproduce and distribute reprints for Governmental purposes notwithstanding any copyright notation thereon.

Any opinions, findings, and conclusions or recommendations expressed in this material are those of the authors and do not necessarily reflect the views, policies, or endorsements, either expressed or implied, of DARPA, NIH, ONR, FDA, or other components of the U.S. Government.

\section{Availability of data and materials}

All data generated or analyzed during this work are included in this published article and its supplementary information files. Supplemental methods and evaluation results are available in "Extracting Chemical Reactions from Text using Snorkel Supplemental Data". The list of 6871 extracted bacterial chemical reaction relationships is available in a separate tab-delimited supplemental file. The supplemental data file contains NCBI PubMed identifiers (i.e., pmids), sentence number, extracted chemicals and character positions in the abstract text, and training marginals for the prediction algorithm. All extracted chemical reaction relationships are predicted as chemical reactions from the discriminative model. Additional data available upon request. We also provide code and labeling functions at http://simtk.org/ projects/chem-rxn

\section{Ethics approval and consent to participate}

Not applicable.

\section{Consent for publication}

Not applicable.

\section{Competing interests}

The authors declare that they have no competing interests.

\section{Author details}

'Biomedical Informatics Training Program, Stanford University, Stanford, CA, USA. ${ }^{2}$ Department of Statistics, Stanford University, Stanford, CA, USA. ${ }^{3}$ Department of Computer Science, Stanford University, Stanford, CA, USA. ${ }^{4}$ Office of Health Informatics, Office of the Chief Scientist, Office of the Commissioner, Food and Drug Administration, Silver Spring, MD, USA. ${ }^{5}$ Departments of Medicine, Genetics, Bioengineering, and Biomedical Data Science, Stanford University, Stanford, CA, USA. 
Received: 3 November 2019 Accepted: 11 May 2020

Published online: 27 May 2020

\section{References}

1. Spanogiannopoulos P, Bess EN, Carmody RN, Turnbaugh PJ. The microbial pharmacists within us: a metagenomic view of xenobiotic metabolism. Nat Rev Microbiol. 2016;14(5):273-87.

2. Caspi R, Billington R, Ferrer L, Foerster H, Fulcher CA, Keseler IM, et al. The MetaCyc database of metabolic pathways and enzymes and the BioCyc collection of pathway/genome databases. Nucleic Acids Res. 2016;44(D1):D471-80

3. Kanehisa M, Goto S. KEGG: Kyoto encyclopedia of genes and genomes. Nucleic Acids Res. 2000;28(1):27-30,

4. Papanikolaou N, Pavlopoulos GA, Theodosiou T, Iliopoulos I. Protein-protein interaction predictions using text mining methods. Methods. 2015;74:47-53.

5. Huang CC, Lu Z. Community challenges in biomedical text mining over 10 years: success, failure and the future. Brief Bioinform. 2016;17(1):132-44.

6. Wei $\mathrm{CH}$, Kao HY, Lu Z. PubTator: a web-based text mining tool for assisting biocuration. Nucleic Acids Res. 2013;41 (Web Server issue):W518-22.

7. Leaman R, Wei CH, Lu Z. tmChem: a high performance approach for chemical named entity recognition and normalization. J Cheminform. 2015;7(Suppl 1 Text mining for chemistry and the CHEMDNER track):S3.

8. Mikolov T, Sutskever I, Chen K, Corrado GS, Dean J. Distributed representations of words and phrases and their compositionality. Advances in neural information processing systems; 2013.

9. Devlin J, Chang M-W, Lee K, Toutanova K. Bert: Pre-training of deep bidirectional transformers for language understanding. arXiv preprint arXiv:181004805; 2018.

10. Lee J, Yoon W, Kim S, Kim D, Kim S, So CH, et al. BioBERT: pre-trained biomedical language representation model for biomedical text mining. arXiv preprint arXiv:190108746. 2019.

11. Krallinger M, Rabal O, Akhondi SA, Pérez MP, Santamaría J, Rodríguez GP, et al. Overview of the BioCreative VI chemicalprotein interaction track. Proceedings of the BioCreative VI challenge evaluation workshop, vol. 2017; 2017.

12. Krallinger M, Rabal O, Leitner F, Vazquez M, Salgado D, Lu Z, et al. The CHEMDNER corpus of chemicals and drugs and its annotation principles. J Cheminform. 2015;7(Suppl 1 Text mining for chemistry and the CHEMDNER track):S2.

13. Ratner A, De Sa C, Wu S, Selsam D, Re C. Data programming: creating large training sets, Quickly. Adv Neural Inf Process Syst. 2016;29:3567-75.

14. Ratner A, Bach SH, Ehrenberg H, Fries J, Wu S, Re C. Snorkel: rapid training data creation with weak supervision. Proceedings VLDB Endowment. 2017;11(3):269-82.

15. Honnibal M, Montani I. spacy 2: Natural language understanding with bloom embeddings, convolutional neural networks and incremental parsing. To appear; 2017.

16. Ratner A, Hancock B, Dunnmon J, Sala F, Pandey S, Ré C. Training complex models with multi-task weak supervision. arXiv preprint arXiv:181002840; 2018

17. Hildebrand A, Schlacta T, Warmack R, Kasuga T, Fan Z. Engineering Escherichia coli for improved ethanol production from gluconate. J Biotechnol. 2013;168(1):101-6.

18. Layh N, Stolz A, Bohme J, Effenberger F, Knackmuss HJ. Enantioselective hydrolysis of racemic naproxen nitrile and naproxen amide to S-naproxen by new bacterial isolates. J Biotechnol. 1994;33(2):175-82.

19. Lee YC, Shlyankevich M, Jeong HK, Douglas JS, Surh YJ. Bioactivation of 5-hydroxymethyl-2-furaldehyde to an electrophilic and mutagenic allylic sulfuric acid ester. Biochem Biophys Res Commun. 1995;209(3):996-1002.

20. Riemenschneider A, Wegele R, Schmidt A, Papenbrock J. Isolation and characterization of a D-cysteine desulfhydrase protein from Arabidopsis thaliana. FEBS J. 2005;272(5):1291-304.

\section{Publisher's Note}

Springer Nature remains neutral with regard to jurisdictional claims in published maps and institutional affiliations.

Ready to submit your research? Choose BMC and benefit from:
- fast, convenient online submission
- thorough peer review by experienced researchers in your field
- rapid publication on acceptance
- support for research data, including large and complex data types
- gold Open Access which fosters wider collaboration and increased citations
- maximum visibility for your research: over 100M website views per year
At BMC, research is always in progress.
Learn more biomedcentral.com/submissions

